\title{
Implementing and Deploying MIB in ATM Transport Network Operations Systems
}

\author{
Tomoaki Shimizu, Ikuo Yoda and Nobuo Fujii \\ NTT Optical Network Systems Laboratories \\ 1-2356 Take, Yokosuka-shi, 238-03 JAPAN \\ E-mail :shimizu@ntttsd.ntt.jp
}

\begin{abstract}
TNMSKernel, a network operations system development platform, can be used to produce a Management Information Base (MIB) in conjunction with a database management system. A previous study used a RDBMS (Relational Database Management System) and an OODBMS (Object-Oriented Database Management System), to implement two functionally equivalent MIB functions. However, these MIB implementations are not suitable for network elements such as digital cross-connect systems and subscriber line terminals. Because processing capabilities including processing power, memories and disk I/O speeds for TMN operations interface attached to them are limited. These problems are solved by implementing a new MIB on the main memory technique. The proposed method offers sufficient performance comparing with methods using RDBMS and OODBMS. Furthermore, this paper describes a strategy of selecting the best MIB implementation for each sub-system in an ATM transport network operations system. The effectiveness of the strategy is confirmed through an experiment on a prototype ATM transport network operations system.
\end{abstract}

\section{Keywords}

MIB, TMN, OSI, Main Memory Resident Database, ATM, Network Element

\section{INTRODUCTION}

The authors have developed an operations system development environment called "TNMSKernel" to efficiently realize transport network operations systems based on TMN (Telecommunications Management Network) standards. The TMN standards provide the operation interface specifications by which multiple carriers can realize telecommunications network interoperability through their operations systems(CCITT M.3010, 1992). The interface specifications utilize the OSI systems management standards, which include the management information model and the common management information services/protocol (CMIS/CMIP) (CCITT X.701, 1992)(CCITT X.711, 1992). TNMSKernel is now being used to develop an ATM transport network operations system (Yata, 1994)(Yoda, 1994). While 
TNMSKernel provides several functions for the rapid development of operations systems, the purpose of this paper is to describe its implementation of MIB (Management Information Base).

As an operations system consists of several sub-systems with different roles, the operational performance of each sub-system determines the total system performance. The performance of each sub-system strongly relies on MIB performance that depends on the MIB implementation and the processing capacity of the sub-system. Two MIB implementations have already been developed and tested on TNMSKernel (Yoda, Sakae, 1992)(Yoda, 1993). Other MIB implementation results can be found in (Dossogne, 1993)(Huslende, 1993). All these studies focused on using RDBMS (Relational Database Management System) or OODBMS (Object-Oriented Database Management System) to implement the MIB function. These approaches seem reasonable only for fairly large sub-systems that we can expect will have the large processing power needed to run the DBMSs. The DBMSs, while they do offer some advantages, impose quite high penalties in terms of computing capacity. Thus, it is difficult to apply the previous implementations to sub-systems with less computing power such as network elements.

What is needed is, therefore, a MIB implementation that is more efficient than the previous approaches. It should produce a MIB that runs quickly, handles all regular management functions, and is suitable for low processing capacity sub-systems.

This paper proposes a MIB implementation that uses the main memory of the managed open system. This realizes a high performance MIB within a limited processing capability. Next, three MIB implementations are evaluated using experimental CMIS operations. Then, the optimal MIB deployment strategy is assessed for the ATM transport network operations system. To do this, the technical requirements of the sub-systems are analyzed. Finally, the effectiveness of the proposed MIB implementation and the deployment strategy is confirmed through an experiment on a prototype system.

\section{REQUIREMENTS FOR MIB IMPLEMENTATION}

\subsection{MIB functions}

MIB consist of managed object instances and their definitions written in the GDMO format (CCITT X.701, 1992)(CCITT X.722, 1992). There are four main MIB functions from the viewpoint of the OSI systems management.

- Management of object instances and attributes: The MIB shall effectively store object instances and attribute values including relationships. It shall also provide a sophisticated information retrieval mechanism.

- Management of containment relationships: Managed object instances named by the containment relationship constitute a tree structure called MIT (Management Information Tree). Since CMIS operations pinpoint the managed object instance to be operated based on this naming rule, the MIB shall map MIT onto MIB.

- Scope and filter: In order for the manager to point to a managed object instance in the managed open system, the base managed object instance, scope and filter parameters are used. The MIB shall have mechanisms to scope managed object instances and filter them by the attribute values specified by the manager.

- Management of transaction: Multiple managing open systems can independently and simultaneously access the same set of management information. Thus, transaction control becomes a critical requirement. The MIB shall ensure the consistency of the management information control functions includes atomic operations for exclusive and transaction controls. 
In addition to the above mentioned functional requirements, the MIB shall have a performance sufficient to handle millions of managed object instances that are spatially distributed. Maintainability and reliability are also required to realize stable network operation.

\subsection{MIB on the platform}

TNMSKernel is the software platform proposed by the authors to develop TMN based operations systems efficiently. TNMSKernel consists of MIB, Human Machine Interfaces, and communication functions including CMIP. These capabilities are supplied as C++ object class libraries (Yoda, 1994). Figure 1 depicts the original TNMSKernel configuration. MIB was originally realized either on a relational database management system(RDBMS) or on an object oriented database management system(OODBMS). Since TNMSKernel provides the MIB Application Program Interface(API), which conceals the database management system's characteristics, programmers can write managed object behavior programs without knowledge of the internal MIB implementation scheme. Mediation programs (Data Base Access API) are used to hide the differences between database management systems. Furthermore, a managed object instance caching mechanism is provided between APIs to improve MIB performance.

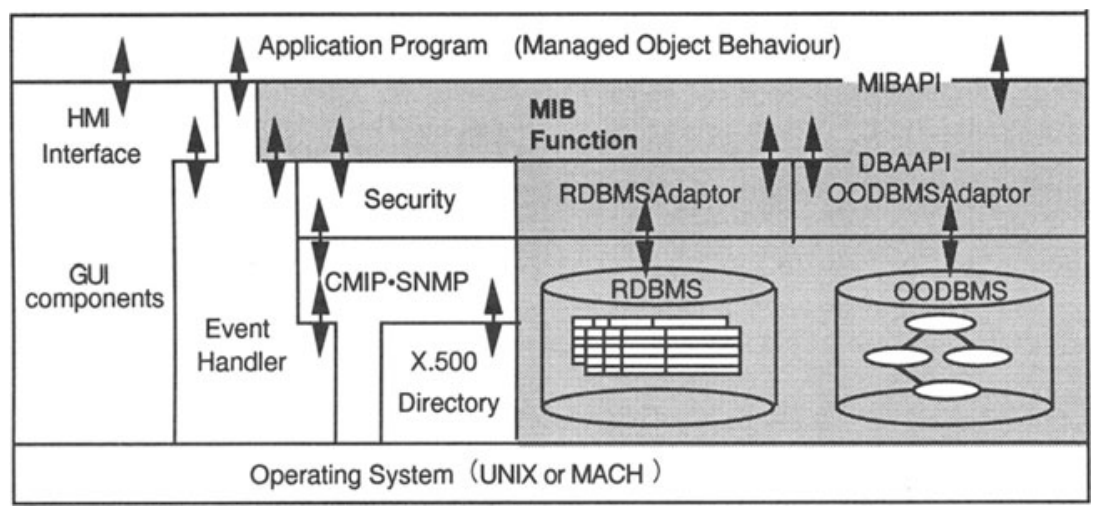

Figure 1 TNMSKernel configuration.

\section{MIB IMPLEMENTATIONS}

TNMSKernel makes use of database management systems to implement MIB functions. The previous research considered only commercially available RDBMS and OODBMS. These are discussed briefly in the following section. Section 3.2 introduces the new idea of Main Memory Resident MIB (MMR-MIB).

\subsection{MIB implementations based on ready-made DBMSs}

\section{$R D B M S$}

RDBMS manages data as tables using mathematical relationships (Ullman, 1988). MIB implementation rules based on an RDBMS are described below (Yoda, 1993). 
- Use an internal object identifier (AOI: Agent Object Identifier) to identify a managed object instance within the MIB.

- Define an attribute table to handle managed object attribute values and define a table key with using an AOI.

- Use a table of AOI pairs to form relationships between containing managed objects and contained managed objects.

- Generate appropriate SQL code from the base object instance, scope and filter parameters specified in the operation request, and perform the management operation.

By mapping managed object instances and attribute values onto RDBMS tables, MIB uses RDBMS as the management information storage tool. To handle managed object instances with the attributes stored in the various relation tables, the table JOIN operation (Ullman, 1988) is needed to perform each management operation. This operation heavily loads the managed system and degrades transaction performance (Yoda, 1993). On the other hand, RDBMS offers an advantage in that the software program is relatively simple but offers powerful scope and filtering procedures. This is because the SQL powerfully supports relationship operations.

\section{OODBMS}

OODBMS permanently stores object instances of complex data structures(Chorafas,1993). This database management system is adequate for the store complex data structures usually found in TMN managed object definitions. The following are the MIB implementation rules for OODBMS implementation.

- Form permanent pointers to indicate managed objects and attributes

- Use pointers to form managed object instance - attribute relationships.

- Realize the containment relationship by having containing and contained object instance pointers in each managed object. The collection management function supported in the OODBMS is used to ensure that the containing managed object has a 1 to $\mathrm{N}$ relationship with contained object instances.

- Find managed object instances using pointer navigation from the base object instances using the conditions specified by scope and filter parameters and perform management operations on them.

The use of OODBMS minimizes the amount of program code needed to realize the MIB function, especially in generating the data schema since managed object instances of complex data structures can be directly stored in the database. Furthermore, pointer processing yields good performance in the management operation on a single managed object instance. By contrast, simultaneous operations on a large number of managed object instances are negatively affected by the clustering effect of object instances on the storage media. Thus, the MIB performance can vary greatly depending on the characteristic of the operation. In addition, the lack of standard query mechanisms such as SQL on RDBMS, increases the amount of program code needed for condition handling.

\subsection{Main Memory Resident MIB}

The OSs (Operating Systems) implemented in most network elements offer only limited capabilities and do not support ready-made DBMS packages. Even if the DBMS is supported, the processors of network elements have relatively low CPU power and low disk I/O speeds. Thus, it is difficult to obtain sufficient processing performance. This means that basing MIB 
on a ready-made database management system, RDBMS or OODBMS, is suitable only when the systems have processing power sufficient to handle large numbers of instances.

What is needed is to replace the ready-made DBMS with another technique; the best candidate is memory resident data handling (Ammann, 1985)(Molina, 1992). It can be used to achieve portable, high performance MIBs that can run on a system with limited CPU power. With this approach, high performance data processing is obtainable by placing and processing managed object instances in the main memory. Figure 2 depicts the concept of the proposed Main Memory Resident MIB (MMR-MIB). This method is configured with the following seven implementation rules.

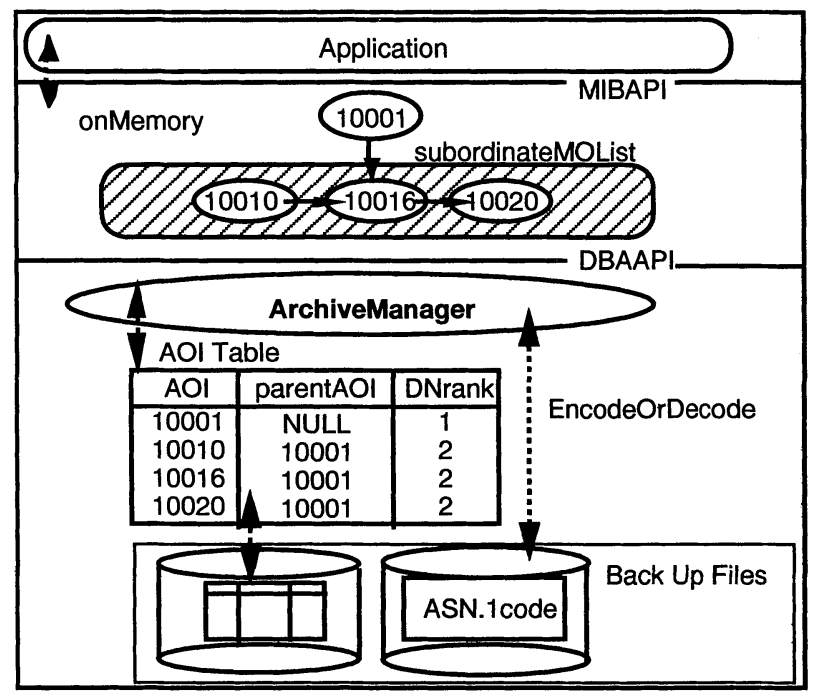

Figure 2 MMR-MIB implementation concept.

- MIB schema: In order to handle managed object instances on the main memory, class definitions themselves are used as the schema information. The class definitions used by application programs are generated from GDMO definitions with using the GDMO translator (Yoda, Minato, 1992).

- Management of managed objects and attributes: The managed object instance is instantiated from the class definition in the MIB schema. Access to the managed object instance and attributes is achieved through the containing managed object pointer and the distinguished attribute.

- Management of containment relationships: The managed object instance has the containing managed object pointer and manages the contained managed object instance pointer group as a unidirectional list. Furthermore, the AOI table is introduced to specify the managed object instance depth on the MIT. This table includes the managed object instance AOI, the containing managed object instance AOI, and the rank of the instance in the MIT.

- Scope and filter: In order to point to the managed object instance, the managed object instance pointing mechanism is furnished. This mechanism processes the logical operators in the scope condition, the filter condition, and AVA(attribute value assertion). 
- Management of transaction: Managed object instance entries including "commit", "abort", "prepare", etc. are employed to realize the atomic operation capability based on two-phase commitment. This capability maintains the data integrity of managed object instances.

- Backup operation: Managed object information is backed-up on nonvolatile memory after the completion of each transaction to avoid the loss of management information. In particular, ArchiveManager object is used to manage AOI table and attribute data encoded in ASN.1 during back-up operations.

- Indexing: The performance of managed object instance access is improved by creating Hash or AVLTree indexes.

\subsection{MIB implementation evaluation}

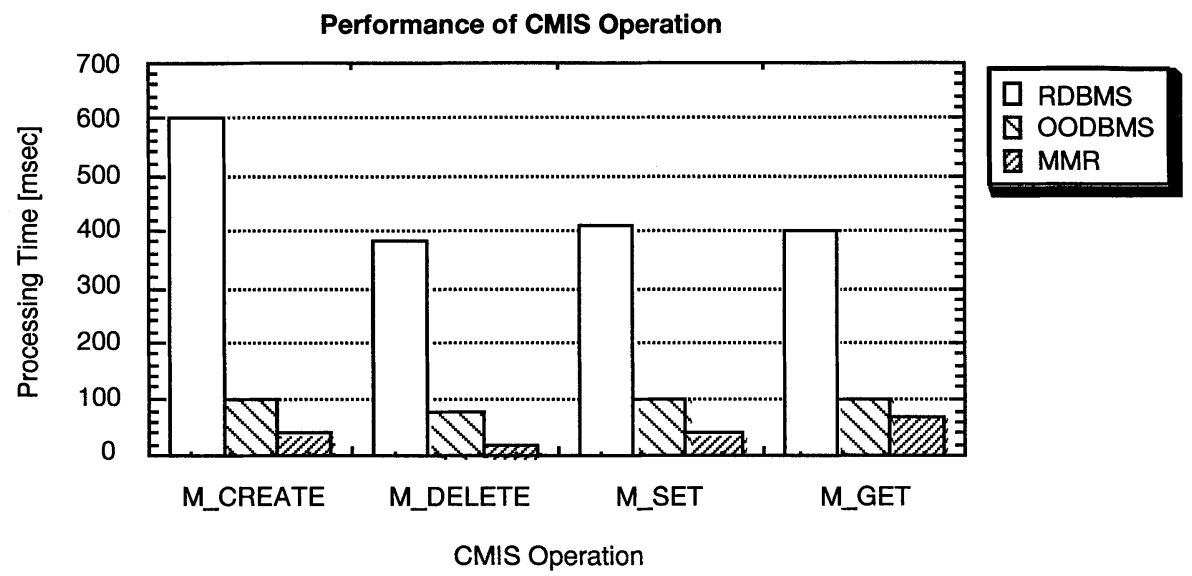

Figure 3 CMIS operations performance.

The above mentioned three implementations were used to implement the same basic MIB, and transaction processing time was measured for each implementation. The MIB, which runs on a UNIX server with RISC processor, stored managed object instances with the attributes of all possible ASN.1 basic data types. Figure 3 shows the average operation times spent to perform CMIS M_CREATE, M_DELETE, M_GET and M_SET operations on a managed object instance as invoked. This result confirms that MMR-MIB achieves the best performance for every examined operation. Regarding M_CREATE operation, MMR-MIB was ten and two times faster that the RDBMS and OODBMS version. In other words, this result shows that MMR-MIB will provide a similar performance to the RDBMS and OODBMS version on control systems that have one tenth and half processing power of RISC processor. While the MMR-MIB implementation supports fewer managed object instances than the other methods, this is not a significant problem for network element applications because network elements manage predictable number of instances and some of them do not require to be persistent in the storage. The performance improvement obtained here is caused by redundant DBMS functions for MIB application elimination such as data schema conversion and ASN.1 data encoding and decoding. 


\section{MIB DEPLOYMENT FOR AN ATM TRANSPORT NETWORK OPERATIONS SYSTEM}

As described in the previous section, each MIB implementation has its advantages and disadvantages. Thus, which is best for each sub-system in a network operation system depends on the technical requirements of the sub-system. This section clarifies sub-system requirements and introduces the strategy of $\mathrm{MB}$ assignment. As an example of a network operation system, let us consider an ATM transport network operations system.

\subsection{System architecture}

Figure 4 depicts the ATM transport network operations system architecture considered in this paper. The hierarchical operations system architecture is adopted (Yoshida, 1992) to increase operation performance and to conform to the TMN standards (CCITT M.3010, 1992). This architecture consists of four layers: the resource layer, the resource control layer, the resource management layer, and the operation scenario management layer. Each layer has sub-systems with MIBs, which store management information to be exchanged through CMIP. The management layers are detailed below.

\begin{tabular}{|c|c|c|c|c|c|}
\hline $\begin{array}{l}\text { Operation } \\
\text { Scenario } \\
\text { Management } \\
\text { Layer }\end{array}$ & \begin{tabular}{|l|} 
End \\
Customer \\
System
\end{tabular} & \multicolumn{2}{|c|}{$\begin{array}{l}\text { Maintenance } \\
\text { Administration } \\
\text { System } \\
\end{array}$} & $\begin{array}{l}\text { Clerk } \\
\text { System }\end{array}$ & \begin{tabular}{l|} 
Construction \\
Administration \\
System
\end{tabular} \\
\hline $\begin{array}{l}\text { Resource } \\
\text { Control } \\
\text { Layer }\end{array}$ & \multicolumn{3}{|c|}{\begin{tabular}{|l|} 
Network \\
Maintenance \\
System
\end{tabular}} & \multicolumn{2}{|l|}{$\begin{array}{l}\text { Network } \\
\text { Construction } \\
\text { System }\end{array}$} \\
\hline $\begin{array}{l}\text { Resource } \\
\text { Management } \\
\text { Layer }\end{array}$ & \begin{tabular}{|l|} 
Network \\
Element \\
Management \\
System \\
\end{tabular} & & $\begin{array}{l}\text { ork } \\
\text { ent } \\
\text { ing } \\
\text { am }\end{array}$ & $\begin{array}{l}\text { Customer } \\
\text { Management } \\
\text { System }\end{array}$ & \begin{tabular}{|l|} 
Workforce \\
Management \\
System
\end{tabular} \\
\hline $\begin{array}{l}\text { Resource } \\
\text { Layer }\end{array}$ & & & $\begin{array}{l}\text { Netwc } \\
\text { Elem }\end{array}$ & & \\
\hline
\end{tabular}

Figure 4 ATM transport network operations system architecture.

1. Resource layer: The sub-systems in the resource layer provide the upper layer sub-systems with a management view of the resources concerned. For example, defects detected by the network element are transformed into alarm notifications. The network element is a potential sub-system in this layer.

2. Resource management layer: The sub-systems in the resource management layer control the management information provided by the resource layer sub-system and generate the management view of logical resources. The network element management system, the network element planning system, the customer management system and the work force management system are located in this layer.

3. Resource control layer: The sub-systems in the resource control layer control the management information of physical and logical resources to provide management views to sub-systems in the operation scenario management layer. Each management view considers 
one component of the management scenario. This layer includes the network maintenance and operations system as well as the network construction system.

4. Operation scenario management layer: The sub-systems in the operation scenario management layer perform management scenarios by controlling the sub-systems of the resource management layer. The end customer control system, the maintenance administration system, the clerk system, and the construction administration system are located in this layer.

\subsection{Managed object model}

Since each sub-system uses CMIP to exchange management information, the management information in each sub-system MIB is modeled as managed objects specified according to GDMO. The managed objects appearing in each management layer are discussed below.

1. Resource layer: As abstractions of network element resources, termination points of SDH Trail and virtual path(VP) Trail, connection information, and packages of equipment and software are modeled as managed objects.

2. Resource management layer: As abstractions of the network, SDH Trails and VP Trails are modeled as managed objects. To handle customer information, the customer's name, the contact phone number, etc. are also modeled.

3. Resource control layer: As components of the management scenario, the procedures used to manage trouble restoration and network construction are modeled as managed objects.

4. Operation scenario management layer: Since sub-system processes of this layer do not play agent roles, there is no managed object except the identifier of agent systems to initiate management operation.

\subsection{Requirements and limitations of systems}

The technical requirements of each layer are described below.

1. Resource layer: A sub-system in this layer is always a managed open system and stores only the management information that represents the components of the sub-system itself. For this reason, it is easy to predict the number of managed objects when designing the system. Moreover, a large storage resource (memory or disk) is not necessary to store these objects. On the other hand, rapid processing is necessary for the sub-systems, particularly for the case of transmitting alarm notification. Another requirement is that the cost of the subsystem should be low. This is because a large number of sub-systems exist in this layer. To satisfy this requirement, the capabilities of the processing machine used in the sub-system must be limited in terms of processing performance attributes such as CPU power or disk I/O speed. For example, existing network elements are using micro-processor based processor machine such as Motorola 68030, Intel 80386 with limited memories less than $128 \mathrm{M}$ bytes.

2. Resource management layer: Sub-systems in this layer must frequently communicate with other sub-systems in other layers and manage a large amount of management information. Therefore, sub-systems must be implemented with server class multipurpose machines.

3. Resource control layer: The requirements are similar to those of the resource management layer; server class multipurpose machines are needed.

4. Operation scenario management layer: Sub-systems in this layer mainly handle the humanmachine interface that allow human operators to access operation functions. This does not require large scale database handling. The multipurpose workstation is probably the best candidate. 


\subsection{MIB deployment strategy}

Table 1 compares the three MIB implementations using the following parameters.

- Processing speed: The processing time needed for CMIP operation.

- Transportability: Program transportability among different machines.

- Software installation cost.

- Storage capacity: The available capacity to store management information.

- System maintainability.

We note that the optimal MIB implementation depends on system-specific requirements. According to the requirements described in Section 4.3, the MIB for sub-systems in the resource management and control layers should be based on ready-made DBMSs (RDBMS or OODBMS) because it needs to handle a lot of management information and facilitate system integration and conversion functions. Meanwhile, sub-systems in the resource layer should adopt MMR-MIB to reduce the implementation cost and to increase the operation performance.

Table 1 Comparison of MIB versions

\begin{tabular}{llll}
\hline Parameters & $R D B M S-M I B$ & $O O D B M S-M I B$ & $M M R-M I B$ \\
\hline processing speed & low & medium & high \\
transportability & no & no & yes \\
software cost & high & high & low \\
storage capacity & large & large & medium \\
maintainability & excellent & good & fair \\
\hline
\end{tabular}

\section{MIB EVALUATION}

\subsection{Evaluation method}

In order to clarify the effectiveness of MMR-MIB in the ATM transport network operations system, a prototype system was developed using the TNMSKernel and evaluated in terms of management processing time. Figure 5 illustrates the target ATM transport network and its management system. The network consists of the ATM cross-connect system(ATM-XC), the ATM subscriber line terminal(ATM-SLT), and the digital subscriber unit(DSU) located in the customer premises. This design has the ATM-SLT manage the DSU while the ATM-SLT and the ATM-XC manage physical resources of the network such as packages and termination points. The network management system (NMS) controls the ATM virtual path (ATM-VP) Trails and the SDH Trails established between network elements. In addition to these components, a debug manager was deployed to initiate the NMS.

In this prototype, the MIB in the NMS was implemented on an OODBMS while the network elements used MMR-MIB. RISC-based UNIX workstations were used as the processing machines of each sub-system in this experiment. The communication protocol between components was CMIP over TCP/IP. The Directory access function was used to realize location transparency of managed objects (Minato, 1993). We examined the following two operation scenarios to evaluate the processing time.

1. SDH Trail Creation: This creates SDH Trail managed objects between ATM-XC and ATMSLT as well as ATM-SLT and DSU. This also creates termination point managed objects such as VP Adaptors, VP connection termination points (VPCTP). 
2. VP Trail Creation: This creates VP Trail from SDH Trails by obtaining the bandwidth of each SDH Trail and establishing the appropriately sized cross-connection in ATM-XC.

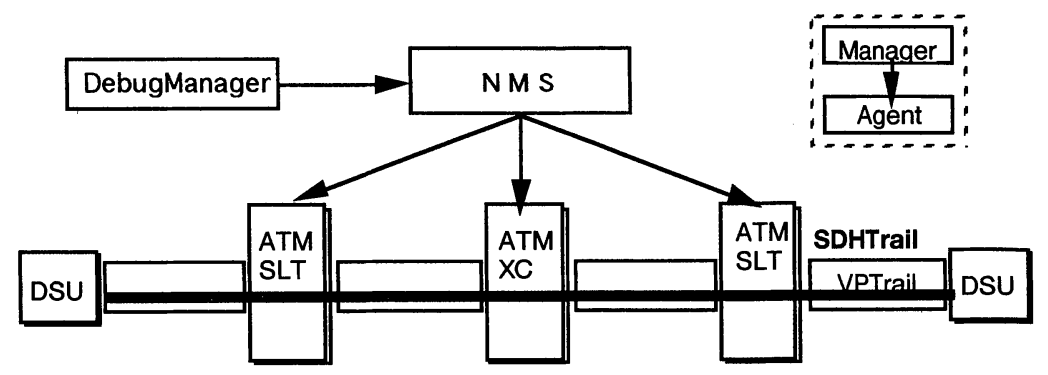

Figure 5 ATM transport network operations system architecture.

\subsection{Evaluation}

A number of CMIP operations made in this experiment are indicated in Table 2 M_GET operations were used to check the availability of network resources. M_SET operations were used to unlock managed object administrative state. M_ACTION operations were used to create multiple VPCTP and cross-connections in SLT and XC while M_CREATE operations were used to create multiple VPCTP of DSU.

Table 2 CMIP operations in the experiment

\begin{tabular}{llcccc}
\hline Operations & agents & $M_{-} G E T$ & $M_{-} S E T$ & $M_{-}$CREATE & $M_{-}$ACTION \\
\hline SDH Trail Creation & NMS & & & 1 & 1 \\
(XC/SLT) & XC & 10 & 4 & 8 & 1 \\
& SLT & 13 & 3 & 8 & 1 \\
\hline SDH Trail Creation & NMS & & & 1 & \\
(SLT/DSU) & SLT(DSU) & 15 & 3 & 260 & \\
\hline VP Trail Creation & NMS & & & 5 & 1 \\
& XC & 3 & & & 1 \\
& SLT(DSU) & 14 & 2 & 2 & 2 \\
\hline
\end{tabular}

Table 3 indicates the average operation processing time for each operation scenario. Managed object creation time for SLT/DSU SDH Trail is large because the SLT manages both termination points on SLT and DSU and it requires 260 VPCTP creations in DSU. The VP Trail Creation time was smaller than SDH Trail Creation time. This is because number of termination point creations are smaller than SDH Trail Creation case. This result verifies that operation performance is sufficient. This operation performance can also be improved by reducing the availability check sequences in each Trail Creation. It also confirms the validity of the proposed MIB deployment strategy. Since a previous experiment on a RDBMS-MIB yield processing times of 10 to 20 seconds(Yata, 1994), the proposed method offers improved operation process time.

Regarding the required MIB size in network elements, it was clarified that an XC needs more than a half million managed object instances to represent its operation function. Thousand managed object instances among them need to be persistent on the storage and less than thirty 
thousand managed object instances need to be visible at the same time. In order to reduce required memory size on XC, we introduced a virtual managed object representation technique to make managed object visible on the MIB memory space when they are needed. Those object instances are reloaded on to the MIB memory by programs. By using this method, it was confirmed that $\mathrm{XC}$ requires less than $70 \mathrm{M}$ bytes memory to realize its operation function. This is within the range of network element processing capability.

Table 3 Operation processing time

\begin{tabular}{lc}
\hline Operations & Average processing time(sec.) \\
\hline SDH Trail Creation (XC/SLT) & 4.3 \\
SDH Trail Creation (SLT/DSU) & 7.5 \\
VP Trail Creation & 3.6 \\
\hline
\end{tabular}

\section{CONCLUSION}

This paper has considered implementing the MIB function on three types of database management systems: RDBMS, OODBMS, and the newly proposed main memory resident technique. The performances of each implementation were evaluated by realizing the same MIB function on "TNMSKernel". The MIB based on the main memory resident technique offers significantly improved performance, which makes it suitable for relatively small systems such as network elements. An MIB deployment strategy was proposed for a hierarchical ATM transport network operations system architecture. Experimental results confirmed that excellent performance is achieved by adopting the appropriate MIB method in each sub-system.

\section{ACKNOWLEDGEMENT}

Authors wish to thank Dr. Ikuo Tokizawa for their support and Dr. Tetsuya Miki for his encouragement. Authors also thank Mr.Kouji Yata, of Telecommunications Software Headquarters, NTT, for his great help in implementing the experimental systems.

\section{REFERENCES}

Ammann, A.C., Hanrahan, M.B. and Krishnamurthy, R. (1985) Design of memory resident DBMS. IEEE COMPCOM.

CCITT Recommendation M.3010 (1992) Principles for a Telecommunications Management Network (TMN).

CCITT Recommendation X.701 (1992) I ISO/IEC 10040 (1992), Information Technology Open Systems Interconnection - Systems management overview.

CCITT Recommendation X.711 (1992) I ISO/IEC 9596-1 (1991 (E)), Information Technology - Open Systems Interconnection - Common Management Information Protocol Specification - Part 1: Specification, Edition 2.

CCITT Recommendation X.722 (1992) I ISO/IEC 10165-4 (1992), Information Technology Open Systems Interconnection - Structure of Management Information: Guidelines for the Definition of managed objects.

Chorafas, D.N. and Steinmann, H. (1993) Object-Oriented Databases, PTR Prentice Hall, Englewood Cliffs,New Jersey. 
Dossogne, F. and Dupont, M.P. (1993) A software architecture for Management Information Model definition, implementation and validation. Integrated Network Management, III(C12), San Francisco.

Huslende, R. and Voldnes, I. (1993) A Q3 Interface for Managing a National Telecommunication Network: Motivation and Implementation. ICC'93, Geneva.

Minato, K., Yoda, I. and Fujii, N. (1993) Distributed Operation System Model using Directory Service in Telecommunication Management Network. GLOBECOM'93, Houston.

Molina, H.G. and Salem, K. (1992) Main Memory Database Systems: An Overview. IEEE Trans. on Knowledge \& Data Engineering 4(6), 509-516.

Ullman, J.D. (1988) Principles of Database and Knowledge-Base systems. Co. Computer Science Press.

Yata, K., Yoda, I., Minato, K. and Fujii,N. (1994) ATM Transport Operation System Based on Object Oriented Technologies. GLOBECOM'94, San Francisco.

Yoda, I., Minato, K. and Fujii, N. (1992) Development of transmission networks operation systems programs by GDMO Translator. Techinical Report of IEICE CS92-54,Japan.

Yoda, I., Sakae, K and Fujii, N. (1992) Configuration of a Local Fiber Optical Network Management System based on Multiple Manager Systems Environment. NOMS'92, Nashville.

Yoda, I. and Fujii, N. (1993) Method for Constructing a Management Information Base (MIB) in Transmission Network Operations: Electronics and Communications in Japan.76, 21-33.

Yoda,I., Yata, K. and Fujii, N. (1994) Object Oriented TMN Based Operations Systems Development Platform. SUPERCOMM/ICC'94, New Orleans.

Yoshida, T., Fujii, N. and Maki, K. (1992) An Object-oriented Operation System Configuration for ATM Networks. ICC'92, Chicago.

\section{BIOGRAPHY}

Tomoaki Shimizu was born in Kanagawa, Japan, in April 1965. In 1988, after receiving his B.S. degree in electronics engineering from Musashi Institute of Technology, Tokyo, Japan, he joined Nippon Telegraph and Telephone Corporation. He has been engaged in the development of private network management systems and currently in the research on transmission network management systems, TMN based operation systems and modeling and implementation of MIB.

Ikuo Yoda was born in Tokyo, Japan, in 1963. He received the B.S. and M.S. degrees in electronics engineering from Waseda University in Tokyo, Japan, 1986 and 1988. In 1988, he joined Nippon Telegraph and Telephone Corporation(NTT's) Transmission systems Laboratories. Since then, he has been engaged in the research on transmission network management systems, TMN based operation systems and modeling and implementation of MIB.

Nobuo Fujii received the B.E. and M.E. degrees in applied physics from Osaka University in 1977 and 1979, respectively. In 1979, he joined NTT. Since then, he has been engaged in the research and development of control system for digital cross-connect systems, the high speed digital leased line system, and the telecommunications network operations system. He is currently running a research group in NTT Optical Network Systems Laboratories. He is a member of the IEEE. 